Brit. J. Ophthal. (1959) 43, 129.

\title{
COMMUNICATIONS
}

\section{AQUEOUS OUTFLOW CHANNELS IN THE TRABECULAR MESHWORK IN MAN*}

\author{
BY \\ JOHN SPEAKMAN† \\ Department of Pathology, Institute of Ophthalmology, University of London
}

MANY anatomical studies of the meshwork have been made in order to define accurately the pathways utilized by the aqueous in reaching the canal of Schlemm. Recent papers confirm the classical division of the trabecular fibres into an inner uveal component extending between the termination of Descemet's membrane and the root of the iris, and an outer corneo-scleral component which joins the innermost corneal lamellae to the scleral spur thus forming the inner wall of the canal. There persists in the literature, however, a fundamental disagreement regarding the nature of the filtration channels adjacent to the canal. There are those, like Ashton, Brini, and Smith (1956), who believe that the perforations in the lamellae continue as far as the wall of Schlemm's canal; and there are others like Flocks (1956), who was unable to demonstrate openings into the canal and therefore suggested the possibility of aqueous "squeezing between the endothelial membranes" surrounding the collagen fibres. The openings in the inner wall of the canal referred to in the past as Sonderman's canals, are too inconstant a feature of the meshwork to be significant, and yet if there are no direct channels communicating with the canal, how can one account for the passage of particulate matter, including red blood cells, from the anterior chamber into the collector channels. The present study of the anatomy of the drainage channels has been made in an attempt to elucidate this problem further.

\section{Material and Methods}

Human eyes containing malignant melanoma, which were rapidly fixed after enucleation and premature baby eyes from 6 months to full-term were used in this investigation. The trabecular region was exposed in the filtration angle by cutting away the iris, and successive layers of fibres were removed with the help of a dissecting microscope and fine forceps and placed beneath a coverslip where they could be stained under direct observation. The inner wall of Schlemm's canal was exposed by making two cuts parallel to Schwalbe's line through the trabeculae into the canal and folding back the fibres thus released. Thin fragments removed from the inner wall of the canal made possible a detailed study of the finer drainage channels which open into its lumen. Additional information was obtained by examining paraffin sections of small blocks of Schlemm's canal which were removed intact from the eye by gross dissection and cut in a variety of

\footnotetext{
*eceived for publication October 13, 1958.
$\dagger$ Wernher Pigott Research Fellow, from the Department of Ophthalmology, University of Toronto. 129
} 
planes. Polychrome methylene blue stain was used for studying cellular detail and the Mallory and Rinehart-Abul-Haj stains were helpful in differentiating the fibre and cellular components.

\section{Uveal Meshwork}

\section{Findings}

The foetal uveal meshwork is incompletely differentiated from the main mass of trabecular cells, but near the iris root the beginnings of the adult pattern can be studied in flat preparations (Figs 1 and 2). They consist of layers of cells disposed in rows around thin bundles of fibres. The cytoplasm of the cells in some places forms a membrane which stretches between the fibres like a diaphragm. These sheets of cytoplasm are thinnest at the centre of the trabecular units and usually one or more oval or round perforations can be seen. As growth proceeds the openings enlarge and the

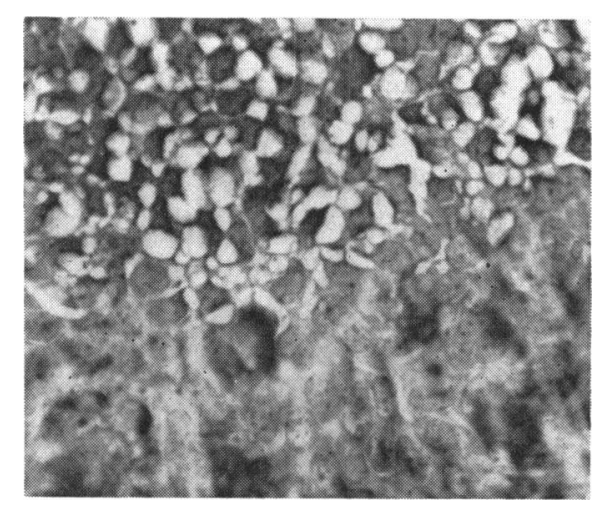

Fig. 1.-Flat preparation, showing abrupt transition from vacuolated corneal endothelium to immature cells and fibres of premature corneo-scleral meshwork. $\times 280$.

(a)
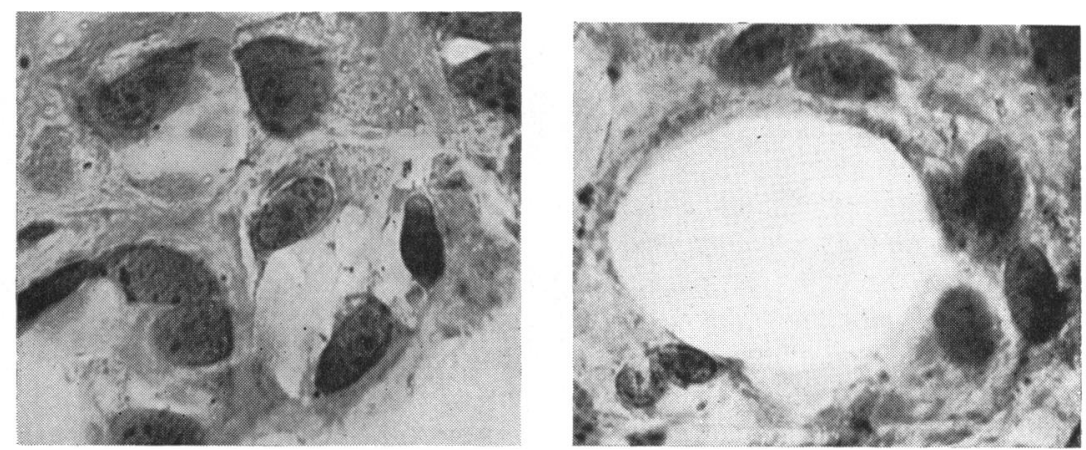

Fig. $2(a, b)$.-Flat preparations, showing different stages in development of openings in uveal meshwork. $\times 630$.

(b) 
membranes of cytoplasm largely disappear. The numerous large ovoid nuclei contain many granular elements and have well-defined nuclear membranes. The fibres at this age consist of many fine collagen bundles which are completely surrounded by cytoplasm. Although the openings in the uveal meshwork are much smaller than in the adult, they appear quite adequate to permit the flow of aqueous into the corneo-scleral meshwork.

The adult uveal meshwork consists of dense bundles of fibres which terminate on one side of the filtration angle in the hyaline substance forming Descemet's membrane and on the other side are firmly attached to the connective tissue of the iris root and to the ciliary muscle (Figs $3 a$ and $b$ ).

(a)

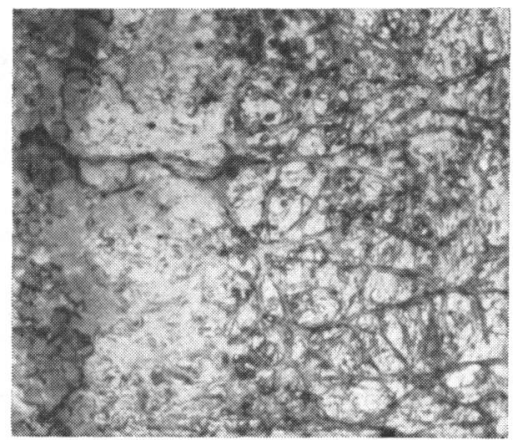

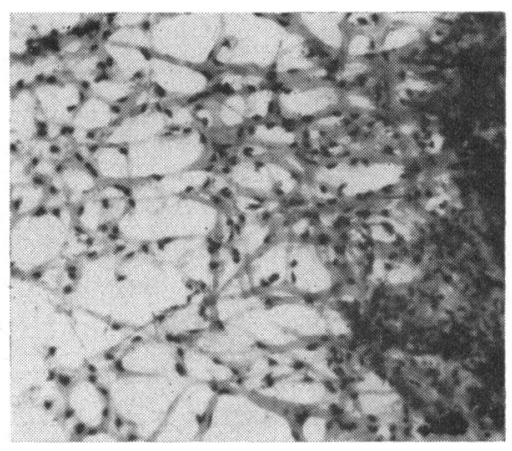

(b)

FIG. $3(a, b)$.-Flat preparation of adult uveal meshwork, showing anterior termination at Schwalbe's line and posterior attachment to root of iris. $\times 60$.

Between these attachments the fibres form a lace-like pattern of large openings and there are numerous connexions with fibres of the adjacent corneoscleral lamellae. Cytoplasm surrounds the fibres in a continuous layer but in places small vacuoles are present. The small darkly-staining nuclei are more dispersed and appear closely applied to the fibres, and the nuclear membrane is less conspicuous than in cells of the premature meshwork. Occasionally, melanophores containing pigment granules in their cytoplasm can be seen encircling the uveal fibres. It is obvious in the adult that aqueous can flow without difficulty through the large openings in the uveal meshwork which lead to the deeper layers.

\section{Corneo-Scleral Meshwork}

The foetal corneo-scleral meshwork is made up of a number of flat lamellae consisting of a syncytium of cells one or two layers in thickness. The cells have large prominent nuclei and between them are formed bundles of fine collagen fibres which are the forerunners of the broad heavy collagen beams seen in the adult eye. Although in flat preparations the tissue may appear solid, one can see in thin areas a basic pattern of large well-defined flat and 
spiral openings in the sheets of cells and fibres which provide intercommunications between adjacent spaces (Figs $4 a$ and $b$ ).

(a)

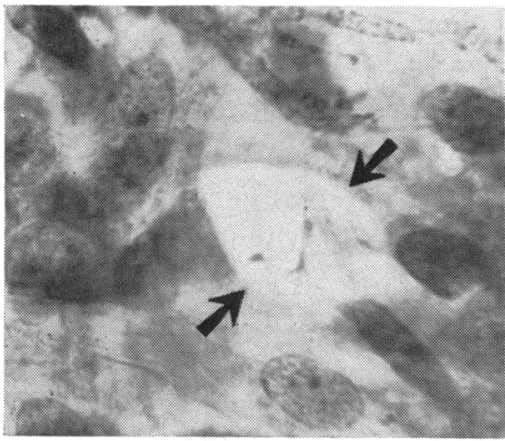

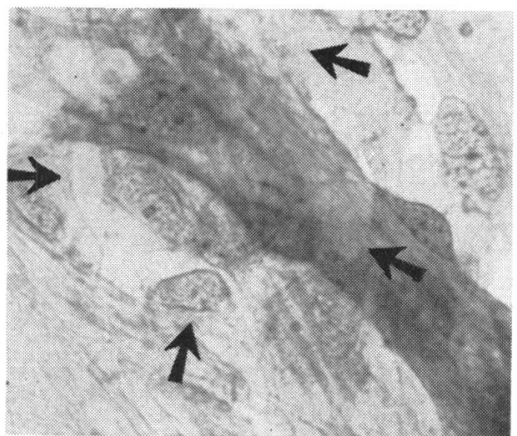

(b)

FIG. $4(a, b)$.-Flat preparations, showing circular and spiral openings in premature corneo-scleral meshwork. $\times 630$.

As growth and differentiation of the eye proceed, more collagen fibrils are synthesised, and eventually an intricate network of interlacing fibres forms which becomes the most conspicuous component of the adult meshwork. It is essential, however, to consider the cellular elements when assessing the calibre of the drainage channels. If in dissecting flat preparations a very thin fragment of tissue is removed, a false impression of the porosity is obtained because most of the cells are pulled away from the fibres (Fig. 5).

FIG. 5.-Flat preparation of adult corneo-scleral meshwork, showing false openings created by removing cellular elements normally present between the clefts. $\times 250$.

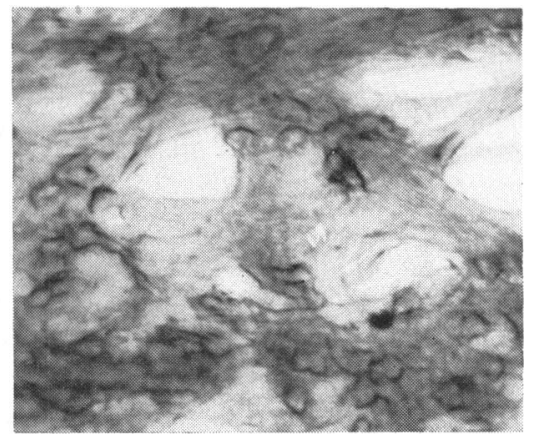

In order to obtain a truer picture it is necessary to examine thicker preparations where the cellular elements are undisturbed. Then it is seen that during the development of the meshwork the layers of cytoplasm remaining in the clefts between the fibres perforate and become drawn into fine strands (Figs 6 and 7, opposite). Thus new small openings form in the lamellae separating the trabecular spaces in addition to those present in the immature eye. These openings can still be recognized by their larger size, their smooth spiral or circular contour, and the absence of cytoplasmic remnants. The simple basic pattern of intercommunication in the premature meshwork is obscured in the adult by this increase in the number and complexity of the openings. 
(a)

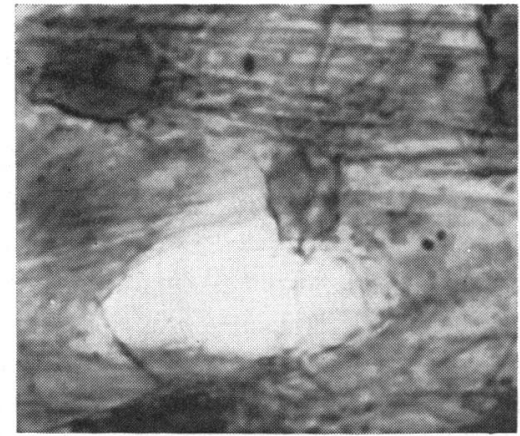

FIG. 6.-Flat preparation of corneoscleral meshwork, showing strands of cytoplasm crossing a cleft and encircling a fibre. $\times 1200$.

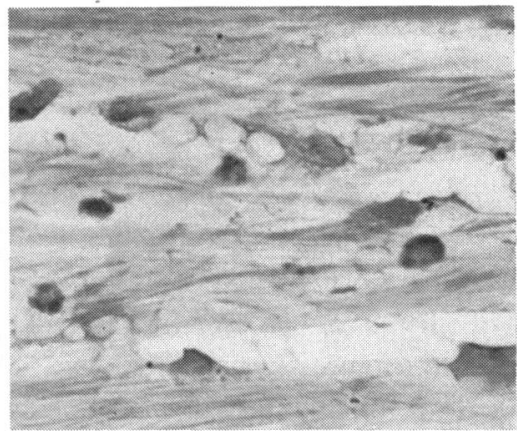

FIG. 7.-Paraffin section, showing strands of cytoplasm in drainage channels and vacuoles in cytoplasm covering fibres. $\times 630$.

The scleral meshwork immediately adjacent to the inner wall of Schlemm's canal is the most interesting and at the same time the most difficult region of the meshwork to visualize. There is a gradual change in the corneo-scleral meshwork as the canal is approached, which is particularly noticeable in the connective tissue component. The thick bands of collagen forming the skeleton of the meshwork become much thinner, and individual collagen fibres can be identified between the thin layers of cytoplasm which line the lamellae. The bundles of fibres which run nearly parallel to the lumen of the canal form a series of troughs and crests, and the clefts between the fibres become long narrow slits. The cells in this region have nuclei with very irregular contours, and many of them which contain pigment granules in their cytoplasm are probably macrophages or melanophores which have wandered through the meshwork from the anterior chamber (Fig. 8).

Fig. 8.-Pleomorphic cells found in meshwork near Schlemm's canal. $\times 840$.

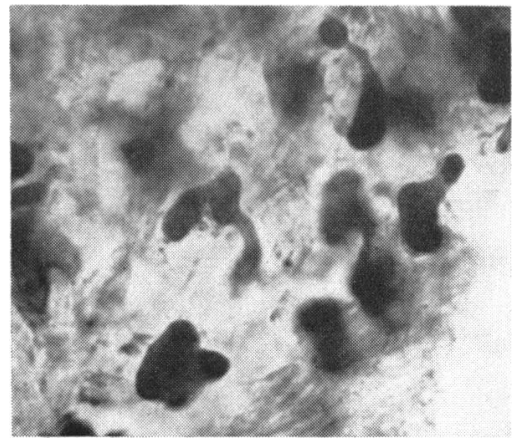

The cytoplasm lining the corneo-scleral meshwork often shows a fine vacuolation, and in some of the cells near the inner wall the vacuoles become quite large making it difficult to assess the porosity, particularly in paraffin sections. Teased preparations show that the drainage pathway consists of a series of intercommunicating channels about $10 \mu$ in width which run obliquely to each other at different levels just inside the wall of the canal, 
and can be traced for distances of 30 to $50 \mu$. The cells in this region, by separating the thin collagen lamellae, maintain the porosity of the meshwork.

\section{Trabecular Wall of Schlemm's Canal}

Flat preparations show that the scleral wall of the canal is relatively smooth but that the trabecular wall has numerous deep grooves in its surface 5 to $20 \mu$ in width and 50 to $100 \mu$ in length (Fig. $9 a$ ). Scattered over the trabecular wall are numerous ovoid and circular openings which lead into the drainage channels in the meshwork (Fig. 9 and Fig. 12,'opposite). Because they do not form a definite pattern it is not possible to determine their total number by scanning a limited region of the inner wall. Some areas are completely devoid of openings whereas in one oil-immersion field seven openings were counted with the following dimensions: $12 \times 6 \mu, 12 \times 5 \mu, 11 \times 8 \mu, 11 \times 7 \mu, 7 \times 2 \mu$, $8 \times 5 \mu$, and $5 \times 4 \mu$. Although the majority of openings are flat, others at the end of a narrow groove resembled the exit from a railway tunnel with dimensions of: $8 \times 5 \mu, 14 \times 5 \mu$, and $5 \times 5 \mu$.

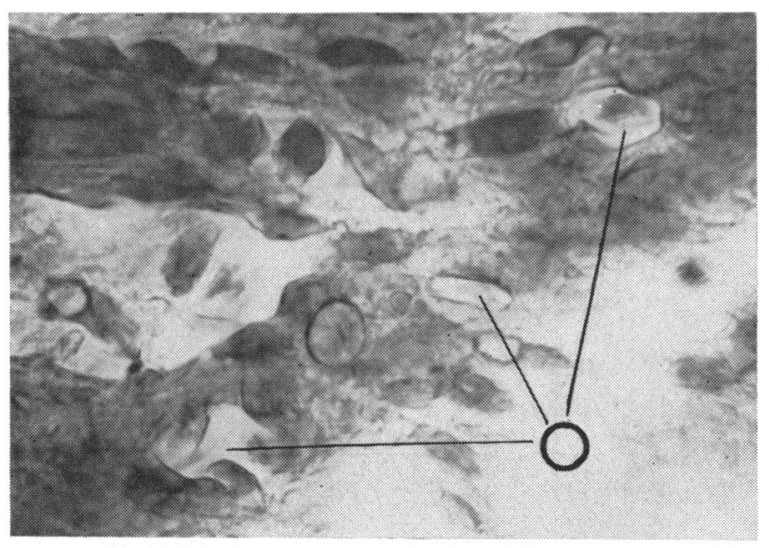

(a)

(b)

FIG. $9(a, b)$.- Flat preparation, showing grooves and openings on trabecular wall of Schlemm's canal. (a) $\times 915 ;(b) \times 1200$.

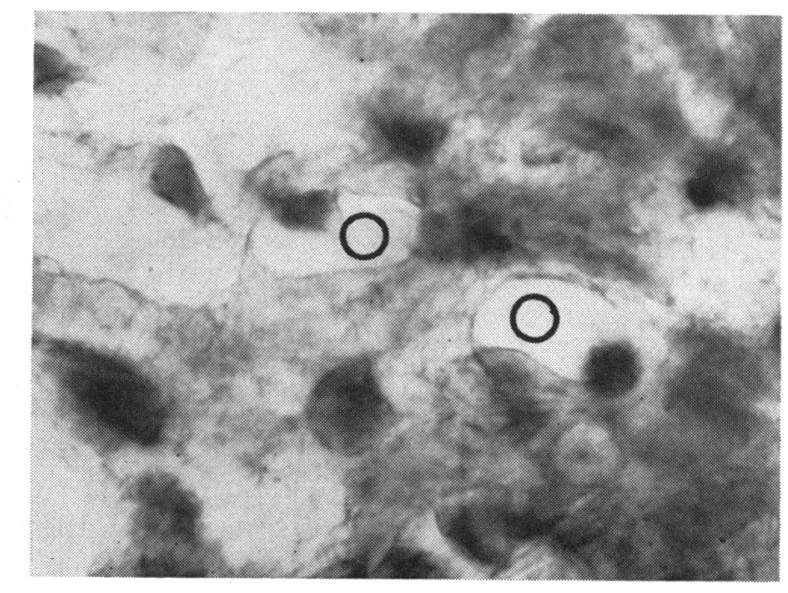


A most interesting feature is the extensive vacuolation present in the cytoplasm lining the trabecular wall (Figs 10 and 11). Near the raised ovoid nuclei where the cytoplasm is thicker, there may be one or two large vacuoles up to $5 \mu$ in diameter. A striking resemblance was noted between the vacuolation in these cells and the vacuolation seen in corneal endothelium,

FIG. 10.-Flat preparation, showing vacuoles in endothelial cells lining inner wall of Schlemm's canal. $\times 950$.
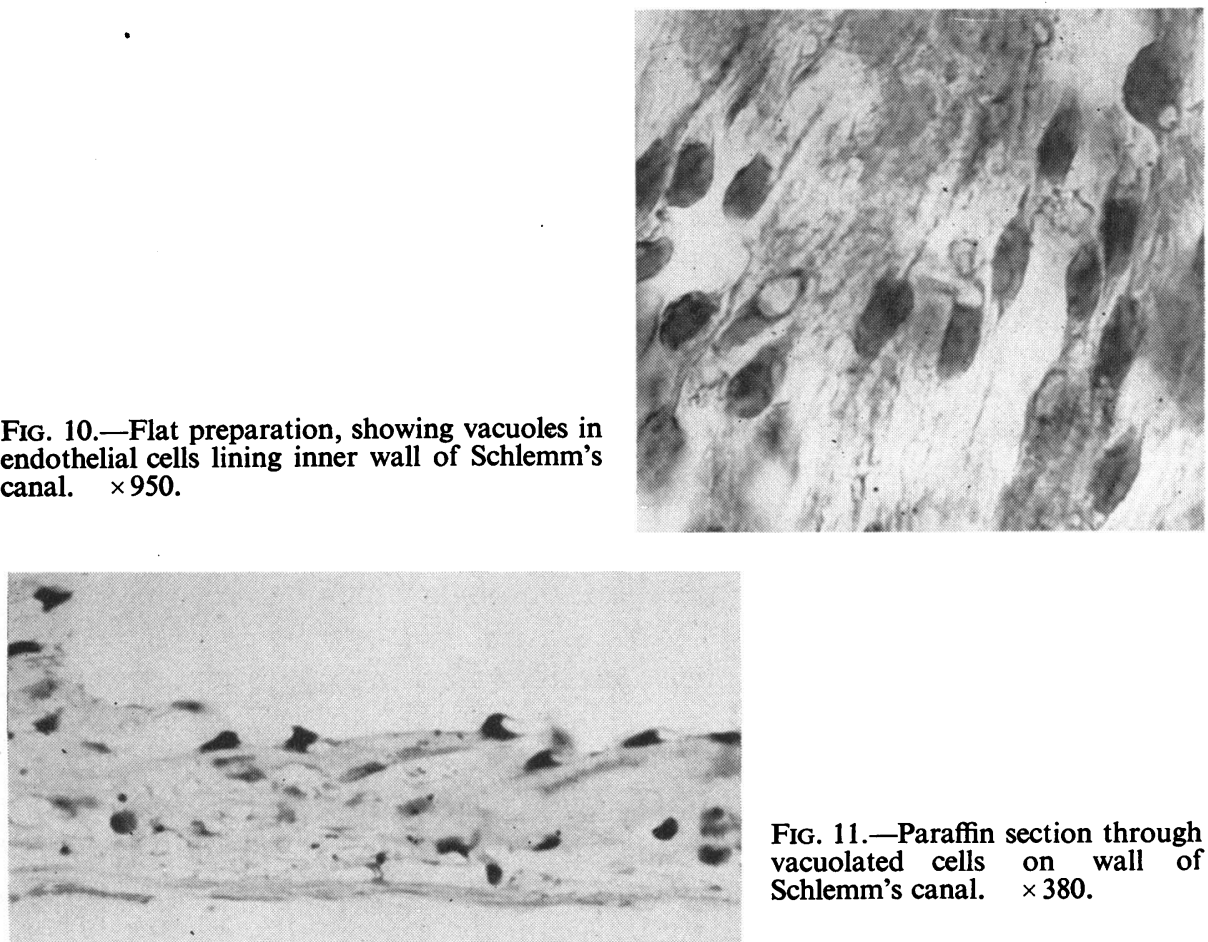

FIG. 11.-Paraffin section through vacuolated cells on wall of Schlemm's canal. $\times 380$.

FIG. 12.-Drawing of openings, grooves, and vacuolated cells on wall of Schlemm's canal illustrated in Figs 9 and $10 . \times 750$.

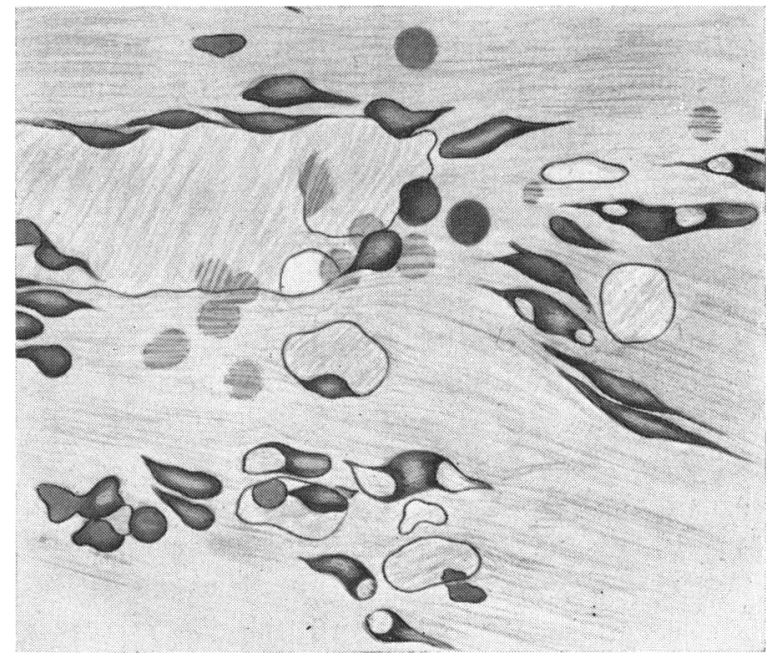


although in the cornea where the cells are packed more closely together the vacuoles are more profuse (Fig. 1). Fresh unfixed endothelium from a rabbit's cornea was therefore studied by phase microscopy and it was found that a similar degree of vacuolation developed very rapidly even though the cells were immersed in warm Tyrode's solution.

Oblique and flat paraffin sections of the canal wall demonstrated the vacuolated nature of the lining cells (Fig. 11), but it was not possible to recognize the openings seen in the flat preparations because of the limitations imposed by the thickness of the sections. Irregularities due to the grooves in the inner wall resembled structures referred to in the literature as Sonderman's canals. The flat preparations showed however that only a few of the smaller grooves, $5 \mu$ in width, opened directly into the meshwork.

\section{Discussion}

A comparison of the openings in the uveal meshwork in adult and premature eyes shows how much the fibres lengthen during development. The growth and differentiation necessary to produce enlargement of the openings also accounts for the alteration in the configuration of the filtration angle with increasing age. No evidence was found to support the concept of a special process of cleavage in the filtration angle which has been postulated by Allen, Burian, and Braley (1955).

The ultimate filtration channels described in this paper support the view that direct communications exist between the anterior chamber and Schlemm's canal. Ashton and others (1956) have previously described stomata in the trabecular wall of the canal and have illustrated one in a flat preparation. However, it seems likely that many of the endothelial lined defects, known as Sonderman's canals, which he and others have illustrated in paraffin sections, are actually grooves on the inner wall of the canal, and are not the true openings, although they may lead to them. The unequivocal demonstration of the openings and grooves on the inner surface of the canal wall should bring to an end the controversy and conflicting reports in the literature regarding the nature of the filtration channels in this region.

The openings themselves are considerably larger than Huggert (1955) and François, Neetens, and Collette (1955) have predicted on the basis of perfusion experiments with particulate matter. Their size, which is approximately $10 \mu$ in diameter, permits red blood cells to penetrate the outer layers of the meshwork without difficulty.

The total number of pores on the inner wall of the canal in a single eye could not be determined accurately, but it is clear from a study of flat preparations that they occur very frequently. The extent of individual variations and the possibility of changes with increasing age are questions which may be answered by further investigation.

At the present time it is difficult to assess the significance of the vacuolated cells on the inner canal wall, which Garron, Feeney, Hogan, and McEwen 
(1958) have also recently demonstrated by electron microscopy of rapidlyfixed human eyes. If these cells have a function similar to that of the corneal endothelium, as their marked tendency to imbibe fluid suggests, it may be that they control the degree of turgescence within the trabecular fibres close to the canal wall.

In view of the extreme narrowing of the drainage channels near Schlemm's canal, it is apparent that only very small alterations in the size of the cells or in the thickness of the collagen fibres would be necessary to alter outflow resistance. Up to the present time there has been little convincing evidence to account for the increased resistance to outflow in chronic simple glaucoma. It may be that pathological obstructions as yet unrecognized begin in the finer drainage channels carrying aqueous through the inner wall of the canal of Schlemm.

The possibility that other cases of chronic simple glaucoma may result from a functional disorder of the trabeculae rather than from primary structural change must also be considered. Any tendency for the lamellae of the corneo-scleral meshwork and the inner wall channels to collapse together would be followed by increased outflow resistance. Conversely, any opening-up of the meshwork should have the reverse effect. Certainly the rapid action of pilocarpine in reducing outflow resistance in cases of openangle glaucoma is evidence against a simple obstruction in the drainage pathway. It appears very likely that pilocarpine and the act of accommodation alter outflow resistance through their action on the trabecular meshwork via the ciliary muscle, but the precise mechanism by which this is brought about must remain speculative until additional evidence is available.

\section{Summary}

(1) A comparison of the trabecular meshwork in the premature and adult eye shows that as development progresses there is an increase in the size and number of openings in the inner lamellae and an increased deposition of collagen in the fibres.

(2) Near the canal of Schlemm the aqueous travels through the meshwork in a series of thin-walled intercommunicating channels which enter the lumen of the canal by means of numerous ovoid openings approximately $10 \mu$ in diameter. The total number of these openings which may be present in a single eye could not be determined accurately, but in one oil-immersion field it was possible to identify seven.

(3) The cells lining the trabecular wall of the canal show a remarkable degree of vacuolation very similar in appearance to the vacuolation which is known to occur in the corneal endothelium as a result of the post-mortem imbibition of fluid. This may indicate a functional similarity between these cells. 
I am greatly indebted to Prof. Norman Ashton for his helpful advice and suggestions during the preparation of this paper. I also wish to thank Mr. B. Fiddy for his valuable technical assistance.

\section{REFERENCES}

Allen, L., Burian, H. M., and Braley, A. E. (1955). A.M.A. Arch. Ophthal., 53, 783.

Ashton, N., BrINI, A., and SMItH, R. (1956). Brit. J. Ophthal., 40, 257.

Flocks, M. (1956). A.M.A. Arch. Ophthal., 56, 708.

François, J., NeEtens, A., and Collette, J. M. (1955). Amer. J. Ophthal., 40, 491.

GarRon, L. K., FeENEY, M. L., Hogan, M. J., and McEwen, W. K. (1958). Ibid., 46, No. 1, pt 2, p. 27.

HUGGerT, A. (1955). Acta ophthal. (Kbh.), 33, 271. 